\title{
Determinación de la resistencia a insecticidas organofosforados, carbamatos y piretroides en tres poblaciones de Anopheles albimanus (Diptera: Culicidae) de Panamá
}

\author{
Lorenzo Cáceres, José Rovira, Arsenio García, Rolando Torres \\ Instituto Conmemorativo Gorgas de Estudio de la Salud, Panamá, República de Panamá
}

\begin{abstract}
Introducción. Se realizó un estudio sobre el estado de la sensibilidad de Anopheles albimanus a insecticidas organofosforados, carbamatos y piretroides, en las localidades de Aguas Claras, Pintupo y Puente Bayano, ubicadas en la comarca indígena de Madugandí, Panamá, región con transmisión de malaria, donde éste es el vector principal.

Objetivo. Determinar el estado de la sensibilidad a organofosforados, carbamatos y piretroides, en tres poblaciones de campo de An. albimanus de Panamá.

Materiales y métodos. Las muestras de la primera generación $\left(\mathrm{F}_{1}\right)$ de $A n$. albimanus recolectadas en las tres localidades de la comarca indígena de Madugandí, fueron expuestas a bioensayos de sensibilidad con insecticidas organofosforados (fenitrotión, malatión y clorpirifos), carbamatos (propoxur) y piretroides (deltametrina, lambdacihalotrina, ciflutrina y cipermetrina), de acuerdo con las normas de la Organización Mundial de la Salud para mosquitos adultos.

Resultados. Las tres cepas de An. albimanus resultaron resistentes a deltametrina, lambdacihalotrina, ciflutrina y cipermetrina, y sensibles a los fenitrotión, malatión, clorpirifos y a propoxur.

Conclusión. Los resultados de esta investigación aportan información importante al programa de control de vectores, y contribuyen al planteamiento de nuevas estrategias sobre el uso de insecticidas, alargando así, la vida útil de los insecticidas en uso.
\end{abstract}

Palabras clave: Anopheles, resistencia a los insecticidas, insecticidas, vectores de enfermedades, insecticidas organofosforados, carbamatos.

Determination of the resistance to organophosphate, carbamate, and pyrethroid insecticides in Panamanian Anopheles albimanus (Diptera: Culicidae) mosquitoes

Introduction. The susceptibility of Anopheles albimanus to organophosphates, carbamates and pyrethroid insecticides was unknown in the Panama communities of Aguas Claras, Pintupo and Puente Bayano, located in the Amerindian Reservation of Madungandi. This region is considered a malaria transmission area, where An. albimanus is the main vector.

Objective. The resistance to organophosphate insecticides, carbamates and pyrethroids was evaluated in field populations of the Anopheles albimanus in Panama.

Materials and methods. Progeny of An. albimanus collected in three localities in the indigenous Madugandi region were exposed to bioassays of susceptibility to organophosphate insecticides (fenitrothion, malathion and chlorpyrifos), the carbamate (propoxur) and pyrethroids (deltamethrin, lambdacyhalothrin, cyfluthrin and cypermethrin). The protocols were in accordance with those established for adult mosquitoes by World Health Organization.

Results. The three strains of the An. albimanus were resistant to the pyrethroid insecticides deltamethrin, lambdacyhalothrin, cyfluthrin and cypermethrin. Susceptibility remained for the organophosphate insecticides fenitrothion, malathion, chlorpyrifos, and the carbamate insecticide propoxur.

Conclusion. The results provided important information to the vector control program, contributing to the application of new strategies on the use of insecticides, and thereby lengthening the life of the insecticide in use.

Key words: Anopheles, insecticide resistance, insecticides, disease vectors; insecticides, organophosphate; carbamates.

Correspondencia:

Lorenzo Cáceres, Instituto Conmemorativo Gorgas de Estudio de la Salud, Apartado postal 0816-02593, Panamá, República de Panamá..

Teléfono: (507) 527 4894; fax: (507) 5274889

Icaceres@gorgas.gob.pa y cacereslorenzo@gmail.com

Recibido: 01/12/10; aceptado:18/06/11
Según la Organización Mundial de la Salud (OMS), a nivel global y a finales del 2004, había zonas de riesgo de transmisión de malaria en 107 países y territorios, y unos 3.200 millones de personas en riesgo de contraer esta enfermedad. Se estima que el número de casos de malaria aumentó de 
233 millones en 2000 a 244 millones en 2005, pero disminuyó a 225 millones en el 2009. El número de muertes por malaria se estima que disminuyó de 985.000 en 2000 a 781.000 en 2009. La disminución en carga de la malaria se ha observado en todas las regiones; proporcionalmente, los mayores descensos se registraron en la región de Europa, seguida por la región de las Américas. Los mayores descensos absolutos de muertes se han observado en África $(1,2)$.

El control de los vectores de importancia médica ha sido parte vital en la estrategia global para el control de los mosquitos incriminados en la transmisión de enfermedades $(3,4)$, y la aplicación de insecticidas representa la medida de intervención práctica más importante y más utilizada para reducir las enfermedades transmitidas por insectos vectores de importancia médica (5). Sin embargo, en la actualidad el incremento considerable de las enfermedades transmitidas por mosquitos ha originado un grave problema de salud pública, debido en parte al desarrollo de resistencia a los insecticidas en las poblaciones de mosquitos $(6,7)$ y de resistencia de los parásitos a los medicamentos antipalúdicos $(8,9)$. El desarrollo de resistencia a los insecticidas en mosquitos vectores de enfermedades como la malaria, es una grave amenaza debido a que una de las principales opciones para la prevención y el control, es el uso de mosquiteros impregnados con insecticidas, el rociamiento de acción residual en los interiores de las viviendas y el uso de larvicidas en criaderos de anofelinos (10). Existe mucha información de la resistencia a los insecticidas en mosquitos vectores de malaria (11).

Anopheles albimanus (Wiedemann, 1820) se considera un vector primario de malaria en las Américas (12). Cuatro de las 10 especies de anofelinos consideradas de importancia en las Américas, han desarrollado resistencia al menos a un insecticida (13). Se ha detectado un alto nivel de resistencia al DDT y bajos niveles de resistencia a insecticidas organofosforados, carbamatos y piretroides, en An. albimanus en México (14). El desarrollo de resistencia a los piretroides en poblaciones de Anopheles es potencial, debido a los tratamientos continuos de rociamiento de las viviendas con este tipo de insecticida y a la impregnación de mosquiteros (15). Los piretroides son actualmente los más ampliamente utilizados para el control de mosquitos en todo el mundo y representan el $25 \%$ de los existentes en el mercado (5).
La problemática de la reemergencia de la malaria en muchas regiones del mundo, está muy estrechamente relacionada al desarrollo de la resistencia a los insecticidas en poblaciones de anofelinos vectores. En India, los insecticidas DDT (organoclorado) y malatión (organofosforado), han estado en uso durante las últimas dos a cinco décadas en el programa de control de vectores y los piretroides sintéticos se introdujeron más tarde para el rociado residual de viviendas en áreas donde los vectores manifestaban resistencia múltiples a insecticidas. Debido al uso constante de estos insecticidas contra las dos principales especies de vectores de la malaria en India, $A n$. culicifacies y An. stephensi, ambas especies han desarrollado resistencia múltiple a insecticidas (16). Los trabajos con vectores de malaria, reportan resistencia al malatión en los principales vectores de malaria, como An. culicifacies, An. stephensi, An. albimanus, An. arabiensis y An sacharovi, a insecticidas piretroides, en An. albimanus, An. stephensi, An. Gambiae, y a carbamatos en $A n$. sacharovi y An. albimanus $(17,18)$.

En Panamá, la malaria siempre ha representado un serio problema de salud pública, debido a su alto costo socioeconómico y es considerada la enfermedad reemergente más importante. En Panamá, al inicio del programa de malaria en 1957, se registraba una tasa de morbilidad elevada, de 765,7 por 100 mil habitantes y una tasa de mortalidad de 18,9, hasta lograr reducir la tasa de morbilidad a 5,8 y la de mortalidad a 0,1, en 1985. A partir del 2002, los casos se duplicaron y se inició un incremento de la malaria, hasta alcanzar en el 20045.097 casos diagnosticados y una tasa de 160,6 por 100 mil habitantes, retrocediendo el comportamiento de la malaria a 35 años atrás.

El análisis de la situación de la malaria en el país durante las últimas décadas, permite establecer la distribución geográfica y los patrones epidemiológicos de la enfermedad, principalmente en regiones con poblaciones indígenas. Durante los últimos años, en estas regiones geográficas es donde se ha diagnosticado más de $85 \%$ del total de los casos a nivel nacional y en ellas habita solamente el $10 \%$ de la población total del país.

Por su parte, el comportamiento de la malaria en la comarca de Madugandí, durante los últimos 10 años (2000 a 2009), registra una tasa promedio de morbilidad de 68,7 por 1.000 habitantes, considerándose esta región como endémica de 
malaria. Los patrones de transmisión en esta región, responden principalmente a factores económicos, socioculturales y de movimientos de población, unidos a los factores biológicos y ecológicos determinantes en la historia de la enfermedad (19). El principal vector de la malaria es An. albimanus, al encontrarse altas densidades de población en las áreas endémicas del país. Entre los anofelinos recolectados en la comarca de Madugandí, están An. albimanus (vector principal de la malaria), An. punctimacula, An. pseudopunctipennis, An. triannulatus, An. malefactor y An. oswaldoi (20).

Desde el inicio del programa de malaria en Panamá, se han utilizado diversos insecticidas organoclorados, organofosforados, carbamatos y piretroides, de forma continua e intensiva, ejerciendo una presión selectiva contra An. albimanus (21). Los estudios con DDT al $5 \%$ en aplicaciones residuales entre 1944 a 1946, en Panamá, demostraron una respuesta modificada de An. albimanus al DDT $(22,23)$. Los estudios con DDT con An. albimanus, de 1944 a 1946 y de 1952 a 1953, presentaron la posibilidad de que se hubiese producido un cambio de conducta intradomiciliaria de An. albimanus, debido al efecto de repelencia por excitación del DDT en la inducción de evasión de superficies rociadas $(24,25)$. En 1972, se llegó a determinar la resistencia fisiológica de An. albimanus al DDT (26). En consideración a la aparición de la resistencia al DDT en An. albimanus, en 1972, el programa de malaria inició el uso del propoxur y, en 1982, del fenitrotión. Entre 1982 y 1988, el programa de malaria llegó a aplicar tres grupos diferentes de insecticidas contra las poblaciones de An. Albimanus: DDT, propoxur y fenitrotión (27). Mediante estudios isoenzimáticos con dos cepas de An. albimanus en 1997, para la detección de niveles elevados de beta-esterasa asociada con la resistencia a insecticidas organofosforados, se logró la detección de niveles moderados de resistencia a fenitrotión en ambas cepas (28). Luego de 25 años de uso del fenitrotión en rociamientos residuales, se remplazó por deltametrina, el cual fue usado hasta el 2002, al detectarse resistencia a este insecticida (29).

Este estudio se hizo para evaluar el estado de la sensibilidad a insecticidas organofosforados, carbamatos y piretroides, en poblaciones de An. albimanus que hubieran estado expuestas a continuas y extensas aplicaciones de insecticidas en tres localidades históricamente endémicas de malaria en Panamá.

\section{Material y métodos}

Sitio de recolección. En este estudio de sensibilidad a insecticidas en anofelinos vectores de malaria, se utilizaron tres cepas de An. albimanus recolectadas en el peridomicilio de las viviendas seleccionadas, en el horario de las 18:00 a las 21:00 horas en las localidades de Aguas Claras ( $9^{\circ} 13^{\prime}$ $10.54^{\prime \prime} \mathrm{N} ; 78^{\circ} 43^{\prime} 01.37^{\prime \prime}$ O y $67 \mathrm{msnm}$ ), Pintupo (9'13' 26.98" N; 78 44' 17.13" O y $74 \mathrm{msnm}$ ) y Puente Bayano ( $9^{\circ} 10^{\prime} 02.38^{\prime \prime}$ N, $78^{\circ} 58.48^{\prime \prime}$ O y 61 msnm), utilizando la metodología recomendada por la OMS para la captura de anofelinos con cebo humano, y siguiendo las recomendaciones técnicas de bioseguridad utilizadas para minimizar el riesgo de transmisión de la malaria en los técnicos de campo (30).

Todo el material biológico de mosquitos adultos recolectado, se colocó en envases especiales debidamente codificados y se transportó a la Sección de Entomología Médica del Instituto Conmemorativo Gorgas, para su identificación a nivel de especie mediante el uso de claves taxonómicas de mosquitos adultos (31). Las cepas de campo de An. albimanus fueron criadas y mantenidas en condiciones de laboratorio, con una temperatura promedio mínima de $28,5{ }^{\circ} \mathrm{C}$ [desviación estándar $(\mathrm{DE})=0,5703$ ) y máxima de $30,0{ }^{\circ} \mathrm{C}(\mathrm{DE}=0,0912)$, humedad relativa de $67,5 \%$ ( $\mathrm{DE}=0,3939)$ y fotoperíodo de 12:12 (día/noche) para, posteriormente, practicar los bioensayos de sensibilidad.

Las tres localidades seleccionadas se encuentran ubicadas en las márgenes del Lago Bayano en el municipio de Chepo, en la comarca de Madugandí. Las localidades estudiadas se seleccionaron con base en la información proporcionada por el Programa Nacional de Malaria del Ministerio de Salud, en cuanto a la magnitud del problema de la malaria y a la necesidad de determinar el comportamiento de la sensibilidad de An. albimanus en esta región del país.

Esta región se caracteriza por ser históricamente palúdica, donde la enfermedad se transmite durante todo el año y ocurre en formas de brotes, con variaciones que están muy asociadas al movimiento de población o migratorio, el clima, los patrones socioculturales, el desarrollo de actividades agrícolas y económicas, el presentar una alta incidencia de la enfermedad por Plasmodium vivax y brotes periódicos por $P$. falciparum durante estos últimos años, aplicaciones frecuentes y continuas 


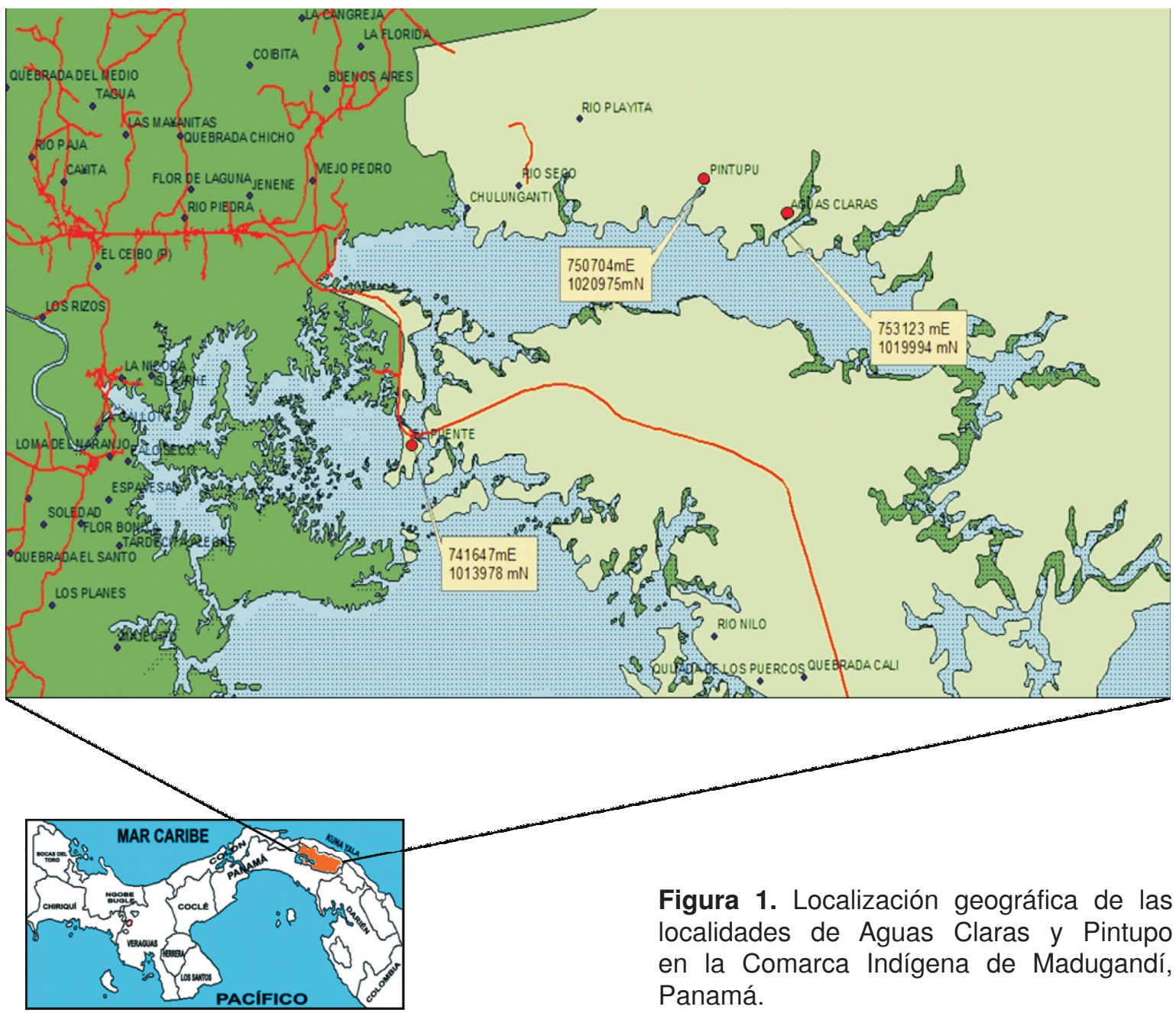

de insecticidas y por ser una comunidad indígena (figura 1). Desde el 2002, el Programa de Malaria hace aplicaciones residuales en las viviendas de las localidades seleccionadas, con fenitrotión y nebulizaciones con deltametrina contra poblaciones de An. albimanus.

Bioensayos de sensibilidad. El comportamiento de la sensibilidad a insecticidas se determinó mediante bioensayos con muestras de hembras adultas de la primera generación $\left(F_{1}\right)$ de las tres cepas de An. albimanus. Las muestras se expusieron a papeles impregnados con los insecticidas piretroides deltametrina $(0,1 \%)$, lambdacihalotrina $(0,1 \%)$, cipermetrina $(0,1$ $\%)$, $\beta$-cipermetrina $(0,1 \%)$, ciflutrina $(0,1 \%)$, los organofosforado fenitrotión (1,0\%) y malatión $(5,0$ $\%)$, y el carbamato propoxur $(0,1 \%)$, con dosis diagnóstica y tiempo de exposición a los insecticidas sugeridas por las normas estandarizadas por la OMS (32). Los papeles impregnados procedían del Instituto de Medicina Tropical "Pedro Kouri" de Cuba, proporcionados por Juan A. Bisset e impregnados de acuerdo con las especificaciones de la OMS.

En los bioensayos se utilizaron lotes de 25 hembras de dos a cuatro días de emergidas y alimentadas con sangre de Cavia porcellus (cobayo); los bioensayos se hicieron una hora después de alimentados los mosquitos. La temperatura promedio registrada durante los bioensayos fue de $27 \pm 2{ }^{\circ} \mathrm{C}$ y $70 \%$ de humedad relativa. Después del período de exposición, los mosquitos se trasladaron a las cámaras de recuperación y se colocó algodón humedecido en solución de glucosa al $10 \%$ como alimento durante el período de recuperación. Se consideró que una mortalidad de 100 a $98 \%$ indica sensibilidad, una de 97 a $80 \%$, que se debe confirmar la resistencia, y una menor de $80 \%$, que hay resistencia $(11,33)$. El tiempo de exposición de los mosquitos a los 
papeles impregnados con insecticidas estuvo de acuerdo con las especificaciones de la OMS. Cada prueba con los insecticidas evaluados contó con cinco réplicas y sus controles respectivos.

Análisis estadísticos. Los porcentajes de mortalidad se registraron a las 24 horas y se sometieron al análisis probit (34). Se tuvo en cuenta la fórmula de Abbott para corregir la mortalidad de los mosquitos expuestos a insecticidas, cuando la mortalidad en los controles osciló entre 5 y $20 \%$ (35).

\section{Resultados}

Como se observa en los cuadros 1, 2 y 3 , las tres cepas de An. albimanus resultaron resistentes a todos los insecticidas piretroides. Los porcentajes de mortalidad se expresaron como medias, con sus respectivas desviaciones estándar.

El menor porcentaje de mortalidad se observó con lambdacihalotrina $(55,6 \% \pm 1,67)$, seguida por cipermetrina $(56,0 \% \pm 2,24)$ en la población de $A n$. albimanus de Aguas Claras; igual comportamiento se pudo constatar con las poblaciones de Pintupo y Puente Bayano.

El mayor porcentaje de mortalidad se registró en la población de An. albimanus de Pintupo $(75,6 \% \pm 1,52)$ con la deltametrina; las otras dos poblaciones mostraron un comportamiento similar, sin diferencias estadísticas significativas.

En cuanto a la exposición de las tres poblaciones de An. albimanus a los insecticidas organofosforados, las tres cepas resultaron sensibles. El porcentaje más bajo de mortalidad se observó en la población de An. albimanus de Aguas Claras con el malatión $(98,6 \% \pm 0,55)$, en tanto que, para fenitrotión y clorpirifos, las poblaciones de An. albimanus de Pintupo y Puente Bayano registraron un porcentaje de mortalidad de $99,0 \%$.

Todos los mosquitos expuestos al propoxur resultaron sensibles a este insecticida con mortalidad de $99,0 \%$. Las tres cepas de An. albimanus fueron persistentemente sensibles a fenitrotión, malatión, clorpirifos y propoxur, sin que se registraran diferencias significativas en el porcentaje de mortalidad.

\section{Discusión}

Mediante este estudio se logró determinar el estado de resistencia a algunos insecticidas piretroides, organofosforados y carbamatos, en la primera generación de hembras adultas de An. albimanus recolectadas en tres localidades de la comarca indígena de Madugandí, región palúdica donde se hacen aplicaciones frecuentes y continuas de diferentes tipos de insecticidas de uso en salud pública. Es importante indicar que, antes de estos estudios, no se habían realizado trabajos recientes en esta región del país.

Los resultados de los bioensayos de sensibilidad mostraron que las tres poblaciones de An. albimanus son resistentes a cipermetrina, lambdacihalotrina, ciflutrina y deltametrina, a los que fueron expuestas.

Cuadro 1. Estado de la resistencia a insecticidas piretroides, organofosforados y carbamatos en adultos de Anopheles albimanus de Pintupo en la comarca indígena de Madugandí, Panamá

\begin{tabular}{|c|c|c|c|c|}
\hline Insecticida & $\begin{array}{l}\text { Mosquitos } \\
\text { expuestos } \\
\text { (n) }\end{array}$ & $\begin{array}{c}\text { Mortalidad } 24 \text { horas } \\
\text { después de la exposición } \\
(\%)^{*}\end{array}$ & $\begin{array}{l}\text { Sobrevivientes } 24 \text { horas } \\
\text { después de la exposición } \\
(\%)^{*}\end{array}$ & $\begin{array}{l}\text { Estado de } \\
\text { la sensibilidad }\end{array}$ \\
\hline Deltametrina & 500 & $\begin{array}{c}378 \\
(75,6 \pm 1,52)\end{array}$ & $\begin{array}{c}122 \\
(24,4)\end{array}$ & Resistente \\
\hline Lambdacihalotrina & 500 & $\begin{array}{c}283 \\
(56,6 \pm 2,07)\end{array}$ & $\begin{array}{c}217 \\
(43,4)\end{array}$ & Resistente \\
\hline Ciflutrina & 500 & $\begin{array}{c}317 \\
(63,4 \pm 2,30)\end{array}$ & $\begin{array}{c}183 \\
(36,6)\end{array}$ & Resistente \\
\hline Cipermetrina & 500 & $\begin{array}{c}279 \\
(55,8 \pm 1,92)\end{array}$ & $\begin{array}{c}221 \\
(44,2)\end{array}$ & Resistente \\
\hline Fenitrotión & 500 & $\begin{array}{c}496 \\
(99,2 \pm 0,84)\end{array}$ & $\begin{array}{c}4 \\
(0,8)\end{array}$ & Sensible \\
\hline Malatión & 500 & $\begin{array}{c}496 \\
(99,2 \pm 0,84)\end{array}$ & $\begin{array}{c}4 \\
(0,8)\end{array}$ & Sensible \\
\hline Clorpirifos & 500 & $\begin{array}{c}496 \\
(99,2 \pm 0,84)\end{array}$ & $\begin{array}{c}4 \\
(0,8)\end{array}$ & Sensible \\
\hline Propoxur & 500 & $\begin{array}{c}496 \\
(99,2 \pm 0,84)\end{array}$ & $\begin{array}{c}4 \\
(0,8)\end{array}$ & Sensible \\
\hline
\end{tabular}

$(\%)^{*}$ : los valores son porcentajes de mortalidad están expresados como media y su desviación estándar. 
Cuadro 2. Estado de la resistencia a insecticidas piretroides, organofosforados y carbamatos en adultos de Anopheles albimanus de Aguas Claras en la comarca indígena de Madugandí, Panamá

\begin{tabular}{|c|c|c|c|c|}
\hline Insecticida & $\begin{array}{l}\text { Mosquitos } \\
\text { expuestos } \\
\text { (n) }\end{array}$ & $\begin{array}{c}\text { Mortalidad } 24 \text { horas } \\
\text { después de la exposición } \\
(\%)^{*}\end{array}$ & $\begin{array}{l}\text { Sobrevivientes } 24 \text { horas } \\
\text { después de la exposición } \\
(\%)^{\star}\end{array}$ & $\begin{array}{l}\text { Estado de } \\
\text { la sensibilidad }\end{array}$ \\
\hline Deltametrina & 500 & $\begin{array}{c}375 \\
(75,0 \pm 1,58)\end{array}$ & $\begin{array}{c}125 \\
(25,0)\end{array}$ & Resistente \\
\hline Lambdacihalotrina & 500 & $\begin{array}{c}278 \\
(55,6 \pm 1,67)\end{array}$ & $\begin{array}{c}222 \\
(35,4)\end{array}$ & Resistente \\
\hline Ciflutrina & 500 & $\begin{array}{c}313 \\
(62,6 \pm 1,52)\end{array}$ & $\begin{array}{c}187 \\
(37,4)\end{array}$ & Resistente \\
\hline Cipermetrina & 500 & $\begin{array}{c}280 \\
(56,0 \pm 2,24)\end{array}$ & $\begin{array}{c}220 \\
(44,0)\end{array}$ & Resistente \\
\hline Fenitrotión & 500 & $\begin{array}{c}495 \\
(99,0-0,55)\end{array}$ & $\begin{array}{c}5 \\
(1,0)\end{array}$ & Sensible \\
\hline Malatión & 500 & $\begin{array}{c}493 \\
(98,6 \pm 0,55)\end{array}$ & $\begin{array}{c}7 \\
(1,4)\end{array}$ & Sensible \\
\hline Clorpirifos & 500 & $\begin{array}{c}497 \\
(99,4 \pm 0,55)\end{array}$ & $\begin{array}{c}3 \\
(0,6)\end{array}$ & Sensible \\
\hline Propoxur & 500 & $\begin{array}{c}498 \\
(99,6 \pm 0,89)\end{array}$ & $\begin{array}{c}2 \\
(0,4)\end{array}$ & Sensible \\
\hline
\end{tabular}

$(\%)^{\star}$ : los valores son porcentajes de mortalidad están expresados como medias y su desviación estándar.

Cuadro 3. Estado de la resistencia a insecticidas piretroides, organofosforados y carbamatos en adultos de Anopheles albimanus de Puente Bayano en la comarca indígena de Madugandí, Panamá

\begin{tabular}{lcccc}
\hline Insecticida & $\begin{array}{c}\text { Mosquitos } \\
\text { expuestos } \\
(\mathbf{n})\end{array}$ & $\begin{array}{c}\text { Mortalidad 24 horas } \\
\text { después de la exposición } \\
(\%)^{*}\end{array}$ & $\begin{array}{c}\text { Sobrevivientes 24 horas } \\
\text { después de la exposición } \\
(\%)^{*}\end{array}$ & $\begin{array}{c}\text { Estado de } \\
\text { la sensibilidad }\end{array}$ \\
\hline Deltametrina & 500 & 378 & 122 & $(24,4)$ \\
Lambdacihalotrina & 500 & $(75,2 \pm 1,52)$ & 217 & Resistente \\
Ciflutrina & 500 & 283 & $(43,4)$ & Resistente \\
Cipermetrina & 500 & $(56,6 \pm 2,07)$ & 183 & Resistente \\
Fenitrotión & 500 & $(63,0 \pm 2,30)$ & $(36,6)$ & Resistente \\
Malatión & 500 & $(56,8 \pm 1,92)$ & $(44,2)$ & Sensible \\
Clorpirifos & 500 & 496 & 4 & $(0,8)$ \\
Propoxur & 500 & $(99,2 \pm 0,84)$ & 4 & Sensible \\
& & 496 & $(0,8)$ & Sensible \\
\hline
\end{tabular}

$(\%)^{\star}$ : los valores son porcentajes de mortalidad están expresados como medias y su desviación estándar.

Entre los piretroides evaluados, se llegó a observar una mayor resistencia a cipermetrina, seguida por lambdacihalotrina, ciflutrina y deltametrina. Por su parte, el propoxur registró un elevado porcentaje de mortalidad en las tres poblaciones de An. albimanus.

La variabilidad observada en la sensibilidad 0 en la resistencia a los insecticidas piretroides, organofosforados y carbamatos en las tres poblaciones de An. albimanus estudiadas, puede deberse a la presión selectiva ejercida por la continua y extensiva aplicación de insecticidas de uso en salud pública y el sector agrícola. En trabajos similares con poblaciones de anofelinos vectores, se observaron variaciones en el comportamiento de la sensibilidad o la resistencia. En Chiapas, en el sur de México, se encontró gran resistencia al DDT y poca resistencia a organofosforados, carbamatos y piretroides, en poblaciones de campo de $A n$. albimanus (36). En poblaciones de An. albimanus 
de Guatemala, se encontró resistencia mostraron a DDT, permetrina y malatión.

Estos resultados indican la importancia de vigilar la resistencia por la aparición de la multirresistencia, especialmente en el campo y a los insecticidas piretroides, y la interacción de los insecticidas utilizados en la agricultura y la salud pública (37). Es importante destacar que los insecticidas piretroides son utilizados en grandes cantidades para las plagas de cultivos. Su uso indiscriminado en la agricultura ha contribuido a la manifestación del problema de resistencia en algunos vectores de malaria (18).

Considerando los resultados de este estudio y con base en trabajos previos, se pone de manifiesto que las poblaciones de An. albimanus de algunos países y territorios de las regiones de las Américas, han desarrollado resistencia a uno o más insecticidas como respuesta al uso histórico y al grado de presión selectiva por largos periodos, debido a las aplicaciones de insecticidas por los programas sanitarios y el uso agrícola.

El problema de la resistencia de An. albimanus, observado en nuestro estudio, se ha comprobado en estudios realizados con el mismo vector en El Salvador, donde detectaron resistencia a propoxur (38). También se ha registrado resistencia en An. vestitipennis de la provincia de Dajabon, en República Dominicana, donde An. albimanus fue resistente a DDT y permetrina, y sensible a malatión, fenitrotión y propoxur. Anopheles vestitipennis fue resistente a DDT y sensible a propoxur (39). En México, se han detectados variaciones similares de la resistencia de An. albimanus al DDT, a insecticidas organofosforados, carbamatos y piretroides (14); igualmente, se ha documentado la resistencia en vectores de paludismo en la costa del Pacífico. Sin embargo, en la península de Yucatán se desconocen los niveles de sensibilidad (40). En Perú, se encontró resistencia a deltametrina en dos cepas de An. albimanus (41).

Esto pone en evidencia que el desarrollo de la resistencia a los insecticidas piretroides en poblaciones de Anopheles es potencial y puede tener un efecto notorio porque puede impactar directamente sobre el control de la malaria, debido a que los piretroides actualmente son muy utilizados en aplicaciones de acción residual a las viviendas y en la impregnación de mosquiteros para el control de vectores (15).

Por otra parte, se ha reportado resistencia a insecticidas carbamatos en An. sacharovi y An.
Albimanus, vectores de malaria (17). Como se puede comprobar, los principales vectores de malaria han desarrollado resistencia a los insecticidas, hecho este que pone de manifiesto la importancia de la vigilancia y seguimiento de la resistencia. A través de trabajos previos se ha demostrado un comportamiento de repelencia por excitación en poblaciones de An. albimanus de El Salvador, Guatemala y Belice a DDT, permetrina y deltametrina, lo que indica que la respuesta de irritabilidad y repelencia a los insecticidas son componentes importantes en las operaciones de control de los vectores de malaria (42).

En cuanto al problema de la resistencia y su impacto en los programas de control de vectores, es importante indicar que se ha argumentado que el problema de la resistencia a los insecticidas sólo debe considerarse como preocupación, cuando llegue a comprometer la efectividad de los insecticidas que son utilizados en las intervenciones realizadas por los programas de control de vectores (43). Considerando este hecho, se puede decir que es una consideración abierta en cuanto a cuál es el nivel de la resistencia que pondría en peligro el control de vectores en los programas. Esto se debe a que los estudios realizados en diferentes áreas han dado resultados variables en cuanto a la resistencia.

En la actualidad, los programas de lucha antivectorial continúan utilizando como herramienta de primera línea a los insecticidas; estos agentes químicos han jugado un papel muy importante en la lucha antivectorial para disminuir o controlar las poblaciones de An. albimanus, con el propósito de prevenir o reducir la transmisión de la malaria en los países de América Latina y, en particular, en nuestro país, donde las epidemias de malaria, han sido básicamente controladas mediante el uso de insecticidas que aun son efectivos contra poblaciones de mosquitos adultos y larvas (44).

Es importante considerar que el desarrollo de la resistencia a los insecticidas es una potencial amenaza para cualquier insecticida que sea utilizado en el control de vectores por los programas de malaria, debido a que el número de insecticidas y formulaciones recomendados por la OMS por el Programa de Evaluación de Plaguicidas (WHOPES) es muy limitado (45).

Es preciso resaltar que estos resultados obtenidos, presentan similitud con otros trabajos previamente realizados con poblaciones de anofelinos vectores de malaria de diferentes regiones de las Américas, África, India y Asia, en los que se han observado 
variaciones en cuanto al comportamiento del estado de la resistencia o de sensibilidad a los insecticidas organofosforados, carbamatos y piretroides. Este hecho, es un indicativo de la gran necesidad de realizar seguimiento permanente que permita llegar a actualizar el comportamiento de la sensibilidad o de la resistencia de las poblaciones de An. albimanus en los diferentes países de las Américas.

Luego de llevar a cabo este trabajo de investigación podemos reafirmar que la vigilancia y el seguimeinto de la resistencia en poblaciones de mosquitos de campo que están sometidas a una presión selectiva de insecticidas, permite lograr detectar focos de poblaciones de insectos tolerantes o resistentes a los insecticidas aplicados por los programas de control de vectores. Entre sus resultados más importantes, es que permite detectar el estado de la sensibilidad o resistencia de forma temprana de las poblaciones de anofelinos vectores que se combaten, facilita la aplicación de medidas de forma oportuna para la prevención del desarrollo de la resistencia, vigila los grados de resistencia en el transcurso del tiempo y confirma si la falta de control, es o no causada por la resistencia.

Los resultados de este estudio aportan información importante al Programa de Control de Vectores del Ministerio de Salud, contribuyendo a la creación de estrategias sobre el uso de insecticidas, de forma tal que se evite el fenómeno de resistencia y así, alargar la vida útil de los insecticidas en uso.

\section{Agradecimientos}

Agradecemos al personal del Departamento de Control de Vectores del Ministerio de Salud, a José García, Bercio Noriega, Santiago Cherigo y León González de la Región de Salud de Panamá Este.

\section{Conflicto de interés}

No hay conflicto de intereses

\section{Financiación}

Esta investigación fue financiada por fondos económicos del Instituto Conmemorativo Gorgas y el apoyo logístico de la Región de Salud de Panamá Este.

\section{Referencias}

1. World Health Organization. Malaria control in complex emergencies. Geneve: World Health Organization; 2005. p. 218.

2. World Malaria Report 2010. WHO Global Malaria Programme. Geneve: World Health Organization; 2009. p. 238.
3. World Health Organization. Expert Committee on Malaria (2000a). 20th Report. WHO Technical Report Series. Geneve: World Health Organization; 2000. p. 892.

4. World Health Organization. The African Summit on Roll Back Malaria (2000c). WHO document WHO/CDS/RBM/2000.17. Geneve: World Health Organization; 2000. p. 241.

5. Casabé N, Melgar F, Wood EJ, Zerba EN. Insecticidal activity of pyrethroids against Triatoma infestans. Insect Sci Applic. 1988;9:233-6.

6. Hemingway J, Hawkes NJ, McCarroll L, Ranson H. The molecular basis of insecticide resistance in mosquitoes. Insect Biochem Mol Biol. 2004;34:653-65.

7. Liu H, Cupp EW, Micher KM, Guo A, Liu N. Insecticide resistance and cross-resistance in Alabama and Florida strains of Culex quinquefasciatus (S.). J Med Entomol. 2004;41:408-13.

8. Phillips RS. Current status of malaria and potential for control. Clin Microbiol Rev. 2001;14:208-26.

9. Hemingway J, Field L, Vontas J. An overview of insecticide resistance. Science. 2002;298:96-7.

10. Hans JO, Simen RS, Suwonkerd W. Evidence of anopheline mosquito resistance to agrochemicals in northern Thailand. Southeast Asian J Trop Med Public Health. 2005;36:152-7.

11. WHO. Expert Committee on Vector Biology and Control Vector Resistance to Pesticides: Fifteenth Report of the WHO Expert Committee on Vector Biology \& Control (WHO technical report series; 818).Geneve: World Health Organization; 1992. p. 62.

12. Fleming G. Biología y ecología de los vectores de la malaria en las Américas. Washington D.C.: OPS/OMS; 1986.

13. Palacios Fraire S. Analysis of the principal problems impeding normal development of malaria eradication programs. Bull Pan Am Health Organ. 1975;9:283-94.

14. Hemingway J, Penilla RP, Rodríguez AD, James BM, Edge W, Rogers H, Rodríguez MH. Resistance management strategies in malaria vector mosquito control. A large-scale field trial in southern Mexico. Pestic Sci. 1997;51:375-82.

15. Hodjati MH, Curtis CF. Evaluation of the effect of mosquito age and prior exposure to insecticide on pyrethroid tolerance in Anopheles mosquitoes (Diptera: Culicidae). Bull Ent Research. 1999;89:329-37.

16. Raghavendra K, Barik TK, Sharma P, Bhatt RM, Srivastava HC, Sreehari U, et al. Chlorfenapyr: a new insecticide with novel mode of action can control pyrethroid resistant malaria vectors. Malar J. 2011;10:1475-80.

17. Hemingway $\mathbf{J}$, Ranson $\mathbf{H}$. Insecticide resistance in insect vectors of human disease. Annu Rev Entomol. 2000;45:371-91.

18. Coleman M, Sharp B, Seocharan I, Hemingway J. Developing an evidence-based decision support system for rational insecticide choice in the control of African malaria vectors. J Med Entomol. 2006;43:663-8.

19. Samudio F, Santamaría AM, Obaldía Nicanor III, Pascale JM, Bayard V, Calzada JE. Prevalence of Plasmodium falciparum mutations associated with antimalarial drug resistance in Kuna Yala, Panama. Am J Trop Med Hyg. $2005 ; 71: 839-41$ 
20. Loaiza JR, Bermingham E, Scott ME, Rovira JR, Conn JE. Species composition and distribution of adult Anopheles (Diptera: Culicidae) in Panama. J Med Entomol. 2008;45:841-51.

21. Cáceres L. La lucha antimalárica en Panamá. Primera edición. Panamá: Editorial Poligrafía, S.A.; 1999. p. 63.

22. Trapido $\mathbf{H}$. The residual of dwellings with DDT in the control of malaria Transmission in Panama, with special reference to Anopheles albimanus. Am J Trop Med Hyg. 1946;26:383415.

23. Trapido H. Modified response of Anopheles albimanus to DDT residual hose spraying in Panama. Am J Trop Med Hyg. 1952;1:853-61.

24. Duret JP. Estudio sobre el comportamiento de los anofelinos del Río Chagres, Panamá. Bol San Panamer. 1961;51:285302.

25. Brown AW, Pal R. Insecticide resistance in arthropods. Monograph series no. 38. Geneve: World Health Organization; 1971. p. 417.

26. Frederikson EC. Bionomics and control of Anopheles albimanus. Technical paper No. 34. Washington, D.C.: Panamerican Health Organization; 1993. p. 76.

27. SNEM. Informe del Programa de Erradicación de la Malaria. Panamá: Ministerio de Salud de Panamá; 1989. p. 66.

28. Cáceres L. Estudio de los niveles de actividad de las esterasas A y B asociadas con la resistencia a insecticidas organofosforados en poblaciones de Anopheles albimanus (Diptera:culicidae) en Panamá. [Tesis de maestría]. Panamá: Universidad de Panamá; 1997. p. 210.

29. Ministerio de Salud de Panamá. Situación de la salud en Panamá. Documento marco. Panamá: Ministerio de Salud de Panamá; 2005. p. 519.

30. Organización Mundial de la Salud. Técnicas entomológicas de campo para la lucha antipalúdica. Ginebra: Organización Mundial de la Salud; 1993. p. 77.

31. Wilkerson RC, Strickman D. Illustrated key to the female anophelinae mosquitoes of Central America and Mexico. $J$ Am Mosq Control Assoc. 1990;6:7-34.

32. World Health Organization. Test procedures for insecticide resistance monitoring in malaria vectors, bio-efficacy and persistence of insecticides on treated surfaces. WHO/CDS/ CPC/ MAL/98.12. Geneva: WHO; 1998. p. 43.

33. World Health Organization. Instructions for determining the susceptibility or resistance of adult mosquitoes to organochlorine, organophosphate and carbamate insecticides. Establishment of the baseline. WHO/ VBC/81.805. Geneva: WHO; 1981.

34. Finney DJ. Probit analysis. 3rd edition. London: Cambridge University Press; 1972. p. 702.

35. Abbott WS. A method for computing the effectiveness of an insecticide. J Economic Entomol. 1925;18:265-7.

36. Penilla RP, Rodriguez AD, Hemingway J, Torres JL, Arredondo-Jimenez JI, Rodriguez MH. Resistance management strategies in malaria vector mosquito control. Baseline data for a large-scale field trial against Anopheles albimanus in Mexico. Med Vet Entomol. 1998;12:217-33.

37. Brogdon WG, McAllister JC, Corwin AM, Cordon Rosales C. Oxidase-based DDT-pyrethroid crossresistance in Guatemalan Anopheles albimanus. Pestic Biochem Physiol. 1999;64:101-11.

38. Ariaratnam V, Georghiou P. Carbamate resistance in Anopheles albimanus. Cross resistance spectrum and stability of resistance. Bull World Health Organ. $1974 ; 51: 655-9$

39. Mekuria Y, Williams DC, Tidwell MA, Santana TA. Studies of the susceptibility of Anopheles albimanus and Anopheles vestitipennis from Dajabon, Dominican Republic, to insecticides. J Am Mosq Cont Assoc. 1990;4:645-50.

40. Dzul FA, Penilla RP, Rodríguez AD. Susceptibilidad y mecanismos de resistencia a insecticidas en Anopheles albimanus del sur de la Península de Yucatán, México. Salud Pública de México. 2007;4:302-11.

41. Vargas F, Córdova O, Alvarado A. Determinación de la resistencia a insecticidas en Aedes aegypti, Anopheles albimanus y Lutzomyia peruensis procedentes del norte peruano. Rev Peru Med Exp Salud Pública. 2006;4:259-63.

42. Chareonviriyaphap T, Roberts DR, Andre RG, Harlan HJ, Manguin S, Bangs MJ. Pesticide avoidance behavior in Anopheles albimanus, a malaria vector in the Americas. $J$ Am Mosq Control Assoc. 1997;13:171-83.

43. Brogdon WG, McAllister JC. Insecticide resistance and vector control. Emerg Infec Dis. 1998;4:605-13.

44. Roberts DR, Andre RG. Insecticide resistance issues in vector-borne disease control. Am J Trop Med Hyg. 1994;50:21-34.

45. World Health Organization. Chemistry and specifications of pesticides. World Health Organ Tech Rep Ser. 2001;899:1-68. 